\title{
Asparagine attenuates hepatic injury caused by lipopolysaccharide in weaned piglets associated with modulation of Toll-like receptor 4 and nucleotide-binding oligomerisation domain protein signalling and their negative regulators
}

\author{
Huanting Wu, Yulan Liu*, Dingan Pi, Weibo Leng, Huiling Zhu, Yongqing Hou, Shuang Li, \\ Haifeng Shi and Xiuying Wang \\ Hubei Collaborative Innovation Center for Animal Nutrition and Feed Safety, Hubei Key Laboratory of Animal Nutrition \\ and Feed Science, Wuhan Polytechnic University, Wuban 430023, People's Republic of China
}

(Submitted 23 November 2014 - Final revision received 27 March 2015 - Accepted 9 April 2015 - First published online 16 June 2015)

\section{Abstract}

Pro-inflammatory cytokines play a key role in many models of hepatic damage. In addition, asparagine (Asn) plays an important role in immune function. We aimed to investigate whether Asn could attenuate lipopolysaccharide (LPS)-induced liver damage. Forty-eight castrated barrows were allotted to four groups including: (1) non-challenged control; (2) LPS-challenged control; (3) LPS + 0.5\% Asn; and (4) LPS $+1.0 \%$ Asn. After $19 \mathrm{~d}$ feeding with control, 0.5 or $1.0 \%$ Asn diets, pigs were injected with LPS or saline. Blood and liver samples were obtained at $4 \mathrm{~h}$ (early stage) and $24 \mathrm{~h}$ (late stage) post-injection. Asn alleviated liver injury, indicated by reduced serum aspartate aminotransferase and alkaline phosphatase activities linearly and quadratically; it increased claudin-1 protein expression linearly and quadratically at $24 \mathrm{~h}$, and less severe liver morphological impairment at 4 or $24 \mathrm{~h}$. In addition, Asn decreased mRNA expression of $T N F-\alpha$ and heat shock protein $70(H S P 70)$ linearly and quadratically at $4 \mathrm{~h}$; it increased $T N F-\alpha$ mRNA expression, and HSP70 protein expression linearly and quadratically at $24 \mathrm{~h}$. Moreover, Asn increased inducible NO synthase activity linearly and quadratically. Finally, Asn down-regulated the mRNA expression of Toll-like receptor 4 (TLR4) signalling molecules (TLR4, IL-1 receptor-associated kinase 1 (IRAK1), TNF- $\alpha$ receptor-associated factor 6), nucleotide-binding oligomerisation domain protein (NOD) signalling molecules (NOD1, $N O D 2$ and their adaptor molecule receptor-interacting serine/threonine-protein kinase 2 (RIPK2)), and $N F-\kappa B p 65$ linearly or quadratically at $4 \mathrm{~h}$. Oppositely, Asn up-regulated mRNA expressions of TLR4 and NOD signalling molecules (TLR4, myeloid differentiation factor 88, IRAK1, NOD2 and RIPK2), and their negative regulators (radioprotective 105, single Ig IL-1R-related molecule, Erbb2 interacting protein and centaurin $\beta 1$ ) linearly or quadratically at $24 \mathrm{~h}$. These results indicate that, in early and late stages of LPS challenge, Asn improves liver integrity and exerts different regulatory effects on mRNA expression of TLR 4 and NOD signalling molecules.

Key words: Asparagine: Hepatic injury: Weaned piglets: Lipopolysaccharide: Toll-like receptor 4: Nucleotide-binding oligomerisation domain protein

Liver, the largest of solid organs in the body, is involved in the crucial detoxification process $^{(1)}$, besides other important functions. In addition, the liver is also an important part of the body's immune response, and is therefore considered an immunological organ ${ }^{(2)}$, which is selectively enriched in macrophages (Kupffer cells). However, multiple factors such as inflammatory stimuli and microbial products, induce Kupffer cells to produce various pro-inflammatory cytokines, such as IL-1 $\beta$, IL-6 and TNF- $\alpha$. Consequently, over-production of these pro-inflammatory cytokines leads to liver injury and liver dysfunction ${ }^{(3,4)}$. Among these cytokines, TNF- $\alpha$ is the central mediator of hepatotoxicity in many models of hepatic damage, especially those involving the lipopolysaccharide (LPS) ${ }^{(5)}$.

Much evidence suggests that innate immunity can specifically detect infection through pattern-recognition receptors

Abbreviations: Asn, asparagines; CONTR, non-challenged control; ERBB2IP, Erbb2 interacting protein; GGT, glutamyl transpeptidase; HSP70, heat shock protein 70; iNOS, inducible NO synthase; IRAK1, IL-1 receptor-associated kinase 1; LPS, lipopolysaccharide; LPSCC, lipopolysaccharide-challenged control; MyD88, myeloid differentiation factor 88; NOD, nucleotide-binding oligomerisation domain protein; NOS, nitric oxide synthase; RIPK2, receptor-interacting serine/threonine-protein kinase 2; RP105, radioprotective 105; SIGIRR, single Ig IL-1R-related molecule; SOCS1, suppressor of cytokine signalling 1; TLR, Toll-like receptor; tNOS, total NO synthase; Tollip, Toll-interacting protein; TRAF6, TNF- $\alpha$ receptor-associated factor 6. 
that recognise pathogen-associated molecular patterns, which are expressed by invading pathogens. Transmembrane Toll-like receptors (TLR) and cytoplasmic nucleotide-binding oligomerisation domain proteins (NOD) are two major forms of innate pattern-recognition receptors ${ }^{(6,7)}$. Among the TLR family, TLR 4 has been detected on all types of liver cells including Kupffer cells, and is activated by a low dose of LPS $^{(8,9)}$. NOD1 and NOD2 were specialised NOD reported to have a direct function as pattern-recognition receptors, in the recognition of peptidoglycan and LPS ${ }^{(10)}$. NOD1 and NOD2 are also highly expressed in liver cells ${ }^{(11,12)}$. Interaction of pathogen-associated molecular patterns with TLR or NOD triggers downstream signalling events that lead to the activation of NF- $\kappa \mathrm{B}$, which then stimulates the expression of inflammatory genes, including TNF- $\alpha$, IL- $1 \beta$ and IL- ${ }^{(6,7)}$. Consequently, the over-expression of pro-inflammatory cytokines elicits collateral host-tissue injury, especially liver injury.

Asparagine (Asn) is a neutral amino acid. Traditionally, Asn is thought as a nutritionally non-essential amino acid in mammals ${ }^{(13,14)}$. However, increasing evidence has shown that Asn plays a significant role in immune function ${ }^{(15)}$ Suzuki et al. ${ }^{(16,17)}$ reported that LPS challenge led to macrophages activation, and it stimulated the production of Asn, $\mathrm{NO}$, citrulline and the consumption of arginine by macrophages. These data suggest the possible link between the activation of macrophages and Asn production, and also suggest a positive relationship between the extent of Asn production and that of NO or citrulline. In the liver, constitutively generated NO maintains the hepatic microcirculation and endothelial integrity, and thereby minimises liver injury ${ }^{(18,19)}$. However, to our knowledge, little research has been conducted to investigate the effect of Asn on liver.

Based on the findings of the studies cited abve, LPS-induced liver injury is characterised by local and systemic inflammation, which is related to Kupffer cell activation via activating TLR4 and NOD signalling pathways ${ }^{(3)}$. So, we hypothesised that Asn might affect TLR 4 and NOD signalling pathways, and exert a beneficial effect on liver integrity. In the present study, we employed a piglet model, an excellent animal model for studying human nutrition and physiology $(20,21)$. The purpose of the present study was to evaluate the effect of dietary Asn supplementation on LPS-induced liver injury, and to elucidate its molecular mechanism(s). Our findings, we hope, will not only help to elucidate the role of Asn in the liver injury of piglets, but also provide new perspectives on developing new nutritional interventions to mitigate human liver damage in inflammatory condition.

\section{Materials and methods}

\section{Pig care and experimental design}

The present study was approved by the Animal Care and Use Committee of Hubei Province, China. A total of forty-eight castrated barrows (Duroc $\times$ Large White $\times$ Landrace; 8.12 (sE 0.56$) \mathrm{kg}$ initial body weight (BW)) were randomly allotted to four groups ( $n$ 12). Pigs were individually caged in $1.80 \times 1.10 \mathrm{~m}$ pens of an environmentally controlled nursery barn. Piglets were allowed ad libitum access to feed and water. The basal diet (see online Supplementary Table S1) was formulated to meet or exceed National Research Council ${ }^{(22)}$ requirements for all nutrients. The amino acid concentration in feeds was analysed according to Wu et al. ${ }^{(23)}$. The room temperature was maintained at $25-27^{\circ} \mathrm{C}$. Lighting was natural.

Treatments included: (1) non-challenged control (CONTR; pigs were fed a control diet and injected with $0.9 \%$ sterile saline); (2) LPS-challenged control (LPSCC; pigs were fed with the same control diet and injected intraperitoneally with Escherichia coli LPS); (3) LPS + 0.5\% Asn treatment (pigs were fed with a $0.5 \%$ Asn diet and injected with LPS); and (4) LPS $+1.0 \%$ Asn treatment (pigs were fed with a $1.0 \%$ Asn diet and injected with LPS). The doses of Asn (L-asparagine, purity > 99\%; Amino Acid Bio-Chemical Company Limited) were used according to our previous study, which showed that dietary supplementation of 0.5 or $1.0 \%$ Asn alleviated weight loss caused by LPS challenge in weanling pigs ${ }^{(24)}$. To obtain isonitrogenous diets, 1.35, 0.68 and $0 \%$ alanine (purity $>99 \%$; Amino Acid Bio-Chemical Company Limited) were added to the control, 0.5 and $1.0 \%$ Asn diets, respectively. After $19 \mathrm{~d}$ feeding with control, 0.5 and $1.0 \%$ Asn diets, the challenged group was injected intraperitoneally with E. coli LPS (E. coli serotype 055:B5; Sigma Chemical, Inc.) at $100 \mu \mathrm{g} / \mathrm{kg} \mathrm{BW}$, and the unchallenged group received an equivalent amount of sterile saline. The dose of LPS $(100 \mu \mathrm{g} / \mathrm{kg}$ BW) was chosen according to our previous studies ${ }^{(3,23,25)}$. After LPS or saline injection, all pigs were fed the same amount of feed $/ \mathrm{kg}$ BW until slaughter in order to avoid the potential effects of LPS-induced feed intake reduction on blood and liver variables. The amount of feed/kg BW was determined according to the feed intake of LPSCC pigs during $24 \mathrm{~h}$ LPS challenge. The pigs were allowed ad libitum access to water.

\section{Blood and liver sample collections}

At $4 \mathrm{~h}$ after LPS or saline injection, six pigs ( $n$ 6) were selected randomly from each treatment group, humanely killed, and blood and liver samples were collected. At $24 \mathrm{~h}$ after LPS or saline injection, the remaining six pigs $(n 6)$ were humanely killed to collect blood and liver samples. The procedures of blood and liver sample collections were according to our previous studies ${ }^{(3)}$. Blood samples were centrifuged $(3500 \boldsymbol{g}$ for $10 \mathrm{~min}$ ) to separate serum which was stored at $-80^{\circ} \mathrm{C}$ until analyses of serum biochemical parameters. One fragment of liver samples was fixed in fresh $4 \%$ paraformaldehyde/PBS at least for $24 \mathrm{~h}$, and embedded in paraffin. The remaining portions were rapidly frozen in liquid $\mathrm{N}_{2}$, and then stored at $-80^{\circ} \mathrm{C}$ for further analysis.

\section{Liver morphology}

Excised liver specimens $\left(0.5 \mathrm{~cm}^{3}\right)$ were fixed in $4 \%$ paraformaldehyde/PBS for $24 \mathrm{~h}$, dehydrated using a graded series of ethanol (70-100\%), cleared with xylene, and embedded in paraffin. Consecutive sections of $5 \mu \mathrm{m}$ thickness were sliced and stained with haematoxylin and eosin for 
microscopic examination. Histological analysis was performed in a blinded manner using a light microscope with a computerassisted morphometric system (BioScan Optimetric; BioScan) by an experienced pathologist.

\section{Serum biochemical parameters}

The activities of serum aspartate aminotransferase (AST), alanine aminotransferase (ALT), glutamyl transpeptidase (GGT) and alkaline phosphatase (AKP) were determined according to Chen et al. ${ }^{(3)}$.

\section{Liver TNF- $\alpha$ concentration and nitric oxide synthase activities}

The TNF- $\alpha$ concentration in liver supernatant was determined using a commercially available porcine ELISA kit (REF: PTA00; R\&D Systems). The minimum detectable dose of TNF- $\alpha$ was $3.7 \mathrm{pg} / \mathrm{ml}$. The result of TNF- $\alpha$ was expressed as $\mathrm{pg} / \mathrm{mg}$ protein. The total NO synthase (NOS) (tNOS) and inducible NOS (iNOS) activities were analysed using a commercially available assay kit (REF: A014-1; Nanjing Jiancheng Biological Product) in accordance with the instructions of the manufacturer. The results of tNOS and iNOS were calculated as $\mathrm{U} / \mathrm{mg}$ protein. One enzyme activity unit (U) was defined as $1 \mathrm{nmol}$ substrate generated per min under the assay conditions.

\section{Western blotting measurement}

The methods for protein immunoblot analysis were according to Chen et al $^{(3)}$. Briefly, hepatic samples (0.15-0.20 g) were homogenised in lysis buffer, and centrifuged to collect the supernatants for Western blot. An equal amount of hepatic proteins $(65 \mu \mathrm{g})$ was loaded onto an SDS-PAGE, and then transferred to polyvinylidene difluoride membranes. The membranes were blocked for at least $60 \mathrm{~min}$ with $5 \%$ non-fat milk in Tris-HClbuffered saline (TBS)/Tween-20 buffer at room temperature, and then incubated overnight $(12-16 \mathrm{~h})$ at $4{ }^{\circ} \mathrm{C}$ with primary antibodies. The membranes were incubated with the secondary antibody for $120 \mathrm{~min}$ at room temperature. Specific primary antibodies included rabbit anti-claudin-1 (REF: 519000, 1:1000; Invitrogen Technology, Inc.), mouse anti-HSP70 (REF: SPA-810, 1:1000; Stressgen) and mouse anti- $\beta$-actin (REF: A2228, 1:10 000; Sigma-Aldrich, Inc.). The secondary antibody included goat anti-rabbit IgG-HRP (REF: ANT020, 1:5000; Antgene Biotech) and goat anti-mouse IgG-HRP (REF: ANT019, 1:5000; Antgene Biotech). Blots were developed using an Enhanced Chemiluminescence Western blotting kit (Amersham Biosciences), and visualised using a Gene Genome bioimaging system. Bands were analysed by densitometry using GeneTools software (Syngene). The relative abundances of claudin- 1 and heat shock protein 70 (HSP70) protein were expressed as claudin-1 protein: $\beta$-actin protein ratio and HSP70 protein: $\beta$-actin protein ratio, respectively.

\section{mRNA expression analysis by real-time $P C R$}

Total RNA isolation, quantification, complementary DNA synthesis and real-time PCR were carried out as previously described $^{(3)}$. The primer pairs used are shown in Table S2 (available online). The expression of the target genes relative to housekeeping gene (glyceraldehyde-3-phosphate dehydrogenase; GAPDH) was analysed by the $2^{-\Delta \Delta C_{\mathrm{T}}} \operatorname{method}^{(26)}$. GAPDH did not exhibit any difference among four treatments. Relative mRNA abundance of each target gene was normalised to the CONTR group

\section{Statistical analysis}

All data were analysed using the general linear model procedure of statistical analysis system appropriate for a factorial arrangement of treatments in a randomised complete block design (SAS Institute Inc.). The statistical model included the effects of treatment (CONTR, LPSCC, LPS $+0.5 \%$ Asn and LPS $+1.0 \%$ Asn), time ( 4 or $24 \mathrm{~h}$ ) and their interactions. Only when a significant treatment $\times$ time interaction occurred, comparisons among treatments in each sampling time ( 4 or $24 \mathrm{~h}$ ) were performed. The following orthogonal contrasts were used to test treatment effects: (1) LPSCC $v$. CONTR was used to determine the response to LPS challenge; (2) linear and quadratic polynomial contrasts were used to determine the response to Asn supplementation among LPS-challenged piglets. Differences were considered as significant at $P \leq 0 \cdot 05$, $0.05<P \leq 0 \cdot 10$ were discussed as trends.

\section{Results}

\section{Liver morphology}

Regarding hepatic morphology, at 4 or $24 \mathrm{~h}$ post-injection, no obvious changes were observed in the livers of the CONTR pigs (Figs. 1(A) and 2(A)). However, morphologic changes associated with liver injury such as hepatocyte caryolysis, karyopycnosis, inflammatory cell infiltration, hepatocyte vacuolisation and hepatic cell cord arrangement in disorder were observed in LPSCC pigs (Figs. 1(B) and 2(B)). Compared with LPSCC pigs, liver injury was attenuated in the LPS $+0.5 \%$ Asn pigs (Figs. $1(\mathrm{C})$ and $2(\mathrm{C})$ ) or LPS $+1.0 \%$ Asn pigs (Figs. 1(D) and 2(D)). Compared with lower concentration ( $0.5 \%)$, higher concentration of Asn (1.0\%) had better protective effect on liver injury.

\section{Serum biochemical parameters}

Significant treatment $\times$ time interactions were observed for serum AST and AKP activities $(P<0.05$; Table 1$)$. At $4 \mathrm{~h}$, compared to the CONTR piglets, LPS challenge increased serum AKP activity $(P<0.05)$.

At $24 \mathrm{~h}$, relative to the CONTR piglets, LPS challenge increased serum AST and AKP activities $(P<0 \cdot 05)$. With increasing Asn supplementation, serum AST and AKP activities were decreased (linear and quadratic, $P<0.01$ ) in LPS-challenged pigs.

No significant treatment $\times$ time interaction was observed for serum ALT, ALT/AST and GGT. Overall, relative to the CONTR piglets, LPS challenge decreased serum ALT:AST ratio $(P<0.05)$, and tended to decrease serum ALT activity $(P=0.093)$. With increasing Asn supplementation, serum ALT activity and ALT:AST ratio were increased (linear and quadratic, 

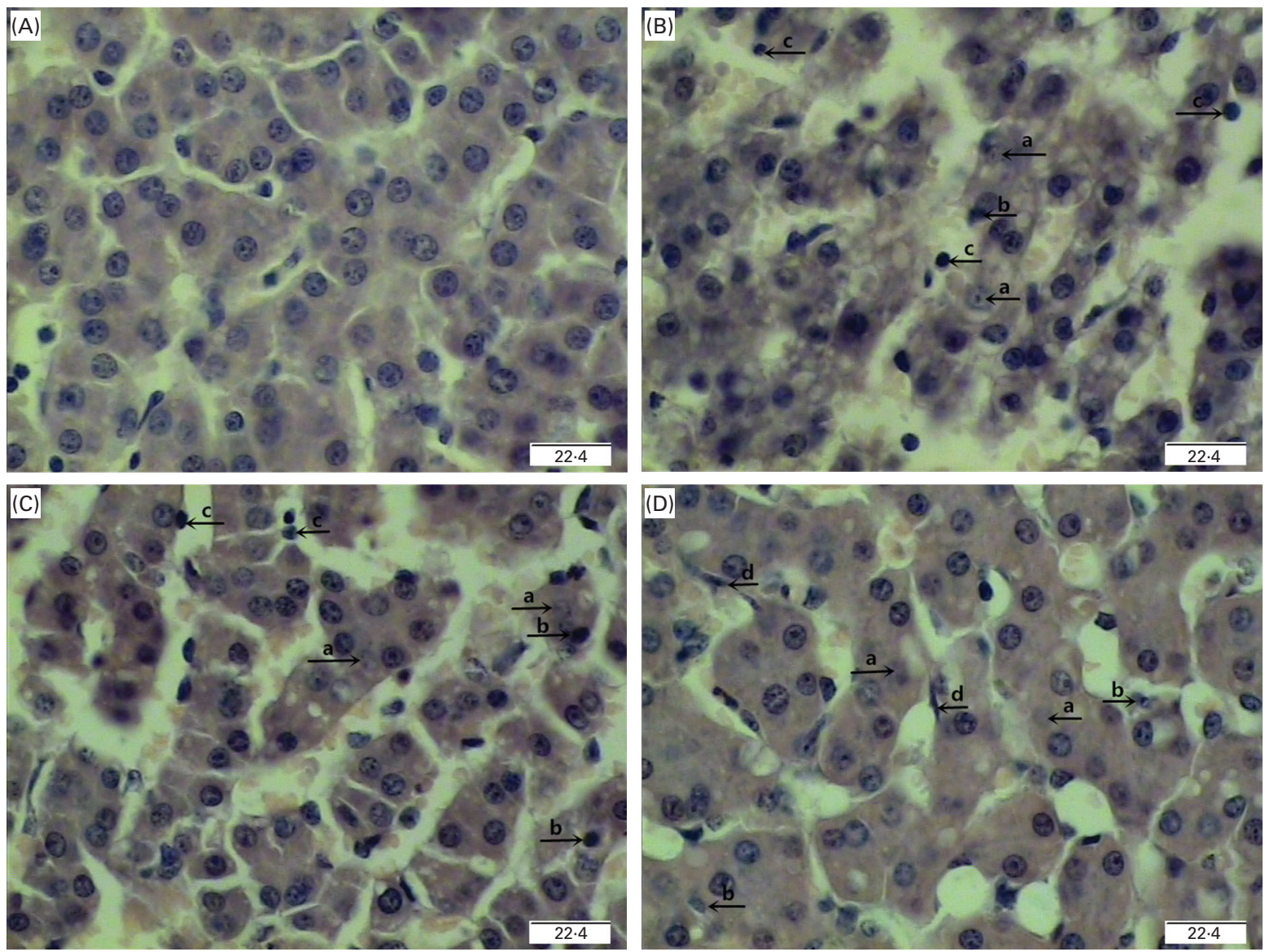

Fig. 1. Effects of asparagine (Asn) supplementation on liver morphology after $4 \mathrm{~h}$ lipopolysaccharide (LPS) challenge in weaned piglets. The representative photomicrographs of liver sections stained with haematoxylin and eosin are shown. (A) Non-challenged control (CONTR; pigs received a control diet and were injected with $0.9 \%$ sterile saline). No obvious changes were found. (B) LPS-challenged control (LPSCC; pigs received the same control diet and were challenged with LPS). Morphologic changes associated with liver injury, such as (a) hepatocyte caryolysis, (b) karyopycnosis, (c) inflammatory cell infiltration, hepatocyte vacuolisation and hepatic cell cords arrangement in disorder, were observed. (C) LPS $+0.5 \%$ Asn treatment (pigs received a $0.5 \%$ Asn diet and were challenged with LPS). (D) LPS + 1.0\% Asn treatment (pigs received a 1.0\% Asn diet and were challenged with LPS), (d) fibroblast proliferation was observed. Liver injury was attenuated in LPS-challenged pigs fed 0.5 or $1.0 \%$ Asn. Compared with $0.5 \%$ Asn, $1.0 \%$ Asn had better protective effect on liver injury. Original magnifications $400 \times$. Scale bars $=22.4 \mu \mathrm{m}$. A colour version of this figure can be found online at $\mathrm{http}: / / \mathrm{www}$.journals.cambridge.org/bjn

$P<0.05)$ in LPS-challenged pigs. Neither LPS nor Asn supplementation had effect on serum GGT activity.

\section{Liver claudin-1 protein expression}

A trend for treatment $x$ time interaction was observed for liver claudin-1 protein expression ( $P=0 \cdot 065$; Fig. 3$)$. At $4 \mathrm{~h}$, relative to the CONTR piglets, LPS challenge decreased liver claudin-1 protein expression $(P<0.05)$.

At $24 \mathrm{~h}$, relative to the CONTR piglets, LPS challenge decreased liver claudin-1 protein expression $(P<0 \cdot 01)$. With increasing Asn supplementation, protein expression of liver claudin-1 was increased (linear and quadratic, $P<0.05$ ) in LPS-challenged pigs.

Liver mRNA and protein abundances of TNF- $\alpha$ and heat shock protein 70

Significant treatment $\times$ time interactions were observed for liver TNF- $\alpha$ and $H S P 7 O$ mRNA abundance $(P<0 \cdot 01$; Table 2$)$, and HSP70 protein abundance $(P<0 \cdot 001$; Fig. 4$)$. At $4 \mathrm{~h}$, compared to the CONTR piglets, LPS challenge increased liver TNF- $\alpha$ and $H S P 7 O$ mRNA abundance, and TNF- $\alpha$ protein concentration $(P<0.05)$. With increasing Asn supplementation, liver TNF- $\alpha$ mRNA abundance (linear and quadratic, $P<0.05$ ) was found decreased, and liver $H S P 70$ mRNA abundance (linear, $P=0 \cdot 067$; quadratic, $P=0.087$ ) and protein abundance (quadratic, $P=0.098)$ tended to be decreased in LPS-challenged pigs.

At $24 \mathrm{~h}, \mathrm{TNF}-\alpha$ protein concentration was undetectable except for the CONTR group. Relative to the CONTR piglets, LPS challenge decreased liver TNF- $\alpha$ mRNA abundance $(P<0.01)$, and tended to decrease liver $H S P 70$ mRNA abundance $(P=0.099)$. With increasing Asn supplementation, liver TNF- $\alpha$ mRNA abundance and HSP70 protein abundance were found increased (linear, $P<0.01$; quadratic, $P<0.05$ ) in LPS-challenged pigs.

Liver total nitric oxide synthase and inducible nitric oxide synthase activities

No significant treatment $\times$ time interactions were observed for liver tNOS and iNOS activities (Table 3). Overall, relative to the 

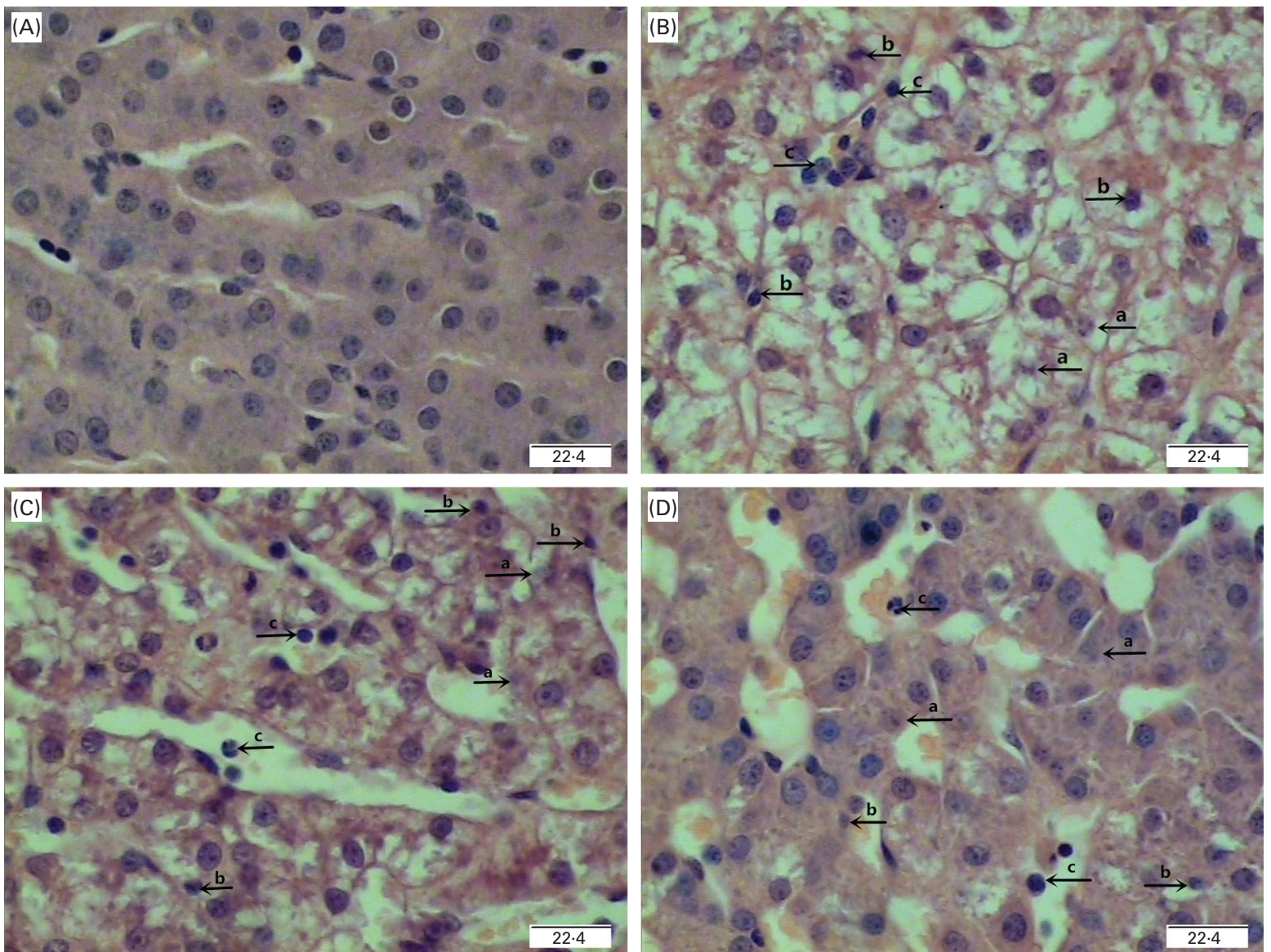

Fig. 2. Effects of asparagine (Asn) supplementation on liver morphology after $24 \mathrm{~h}$ lipopolysaccharide (LPS) challenge in weaned pigs. The representative photomicrographs of liver sections stained with haematoxylin and eosin are shown. (A) Non-challenged control (CONTR; pigs received a control diet and were injected with $0.9 \%$ sterile saline). No obvious changes were found. (B) LPS-challenged control (LPSCC; pigs received the same control diet and were challenged with LPS). Morphologic changes associated with liver injury, such as (a) hepatocyte caryolysis, (b) karyopycnosis, (c) inflammatory cell infiltration, hepatocyte vacuolisation and hepatic cell cords arrangement in disorder, were observed. (C) LPS $+0.5 \%$ Asn treatment (pigs received a $0.5 \%$ Asn diet and were challenged with LPS). (D) LPS + 1.0\% Asn treatment (pigs received a 1.0\% Asn diet and were challenged with LPS). Liver injury was attenuated in LPS-challenged pigs fed 0.5 or $1.0 \%$ Asn. Compared with $0.5 \%$ Asn, $1.0 \%$ Asn had better protective effect on liver injury. Original magnifications $400 \times$. Scale bars $=22.4 \mu \mathrm{m}$. A colour version of this figure can be found online at http://www.journals.cambridge.org/bjn

CONTR piglets, LPS challenge decreased liver iNOS activity $(P<0.05)$. With increasing Asn supplementation, liver iNOS activity was found increased (linear, $P<0.05$; quadratic, $P=0.054)$ in LPS-challenged pigs. Neither LPS nor Asn supplementation had any effect on liver tNOS activity.

Liver mRNA expression of Toll-like receptor 4 and nucleotide-binding oligomerisation domain protein signalling and their downstream signals

Significant treatment $\times$ time interactions were found for mRNA abundance of liver TLR4, myeloid differentiation factor 88 (MyD88), IL-1 receptor-associated kinase 1 (IRAK1), TNF- $\alpha$ receptor-associated factor 6 (TRAFO), NOD1, NOD2, receptor-interacting serine/threonine-protein kinase 2 (RIPK2) and $N F-\kappa B$ p 65 ( $P<0 \cdot 01$; Table 4$)$. At $4 \mathrm{~h}$, relative to the CONTR piglets, LPS challenge increased mRNA abundance of liver TLR4, MyD88, IRAK1, NOD1, NOD2, RIPK2 and NF-кB p65 $(P<0 \cdot 01)$, and tended to increase mRNA abundance of liver TRAF6 $(P=0.056)$. With increasing Asn supplementation,
mRNA abundance of liver TLR4, IRAK1, TRAFG, NOD1, $N O D 2$ and $N F-\kappa B p 65$ were found decreased (linear and quadratic, $P<0.05$ ), and RIPK2 tended to be decreased (quadratic, $P=0 \cdot 088)$ in LPS-challenged pigs.

At $24 \mathrm{~h}$, relative to the CONTR piglets, LPS challenge decreased mRNA abundance of liver TLR4, MyD88, IRAK1, NOD2 and RIPK2 $(P<0.05)$, and tended to decrease mRNA abundance of liver TRAFG $(P=0.089), N O D 1(P=0.088)$ and $N F-\kappa B$ p65 ( $P=0.074)$. With increasing Asn supplementation, mRNA abundance of liver TLR4, MyD88, IRAK1, TRAFG, NOD 2 and RIPK2 were found increased linearly and quadratically $(P<0.05)$, and NOD1 was found increased linearly $(P<0.05)$ in LPS-challenged pigs.

Liver mRNA expression of negative regulators of Toll-like receptor 4 and nucleotide-binding oligomerisation domain protein signalling pathways

Significant treatment $\times$ time interactions were found for mRNA abundance of liver radioprotective 105 (RP105), suppressor of 
cytokine signalling 1 (SOCS1), single Ig IL-1R-related molecule $($ SIGIRR $) \quad(P<0 \cdot 01)$, Erbb2 interacting protein $(E R B B 2 I P)$ $(P=0.056)$ and centaurin $\beta 1$ (CENTB1) $(P=0.062)$ (Table 5). At $4 \mathrm{~h}$, compared to the CONTR piglets, LPS challenge increased mRNA abundance of liver SOCS1, and decreased mRNA abundance of liver SIGIRR and CENTB1 $(P<0 \cdot 05)$.

At $24 \mathrm{~h}$, relative to the CONTR piglets, LPS challenge decreased mRNA abundance of liver RP105, SOCS1, ERBB2IP and CENTB1 $(P<0.05)$, and tended to decrease mRNA abundance of liver SIGIRR $(P=0.099)$. With increasing Asn supplementation, mRNA abundance of liver $R P 105$ (linear, $P<0.01$; quadratic, $P<0.05$ ), SIGIRR (linear, $P<0.01$; quadratic, $P<0.05$ ), ERBB2IP (linear, $P<0.05$; quadratic, $P=0.083$ ) and CENTB1 (linear, $P<0.05$; quadratic, $P=0.054$ ) were found increased, and SOCS1 tended to be increased (linear, $P=0.069)$ in LPS-challenged pigs.

No significant treatment $\times$ time interaction was observed for liver Toll-interacting protein (Tollip) mRNA abundance. Overall, compared to the CONTR pigs, LPS challenge decreased liver Tollip mRNA abundance $(P<0 \cdot 01)$. With increasing Asn supplementation, mRNA abundance of liver Tollip was found increased (linear, $P<0.05$; quadratic, $P=0.084$ ) in LPS-challenged pigs.

\section{Discussion}

The intraperitoneal injection of LPS is widely used for the study of liver injury ${ }^{(3,4,25)}$. As the integral component of the cell wall of Gram-negative bacteria, LPS is prototypical example of endotoxin ${ }^{(4,27)}$. Substantial evidence has shown

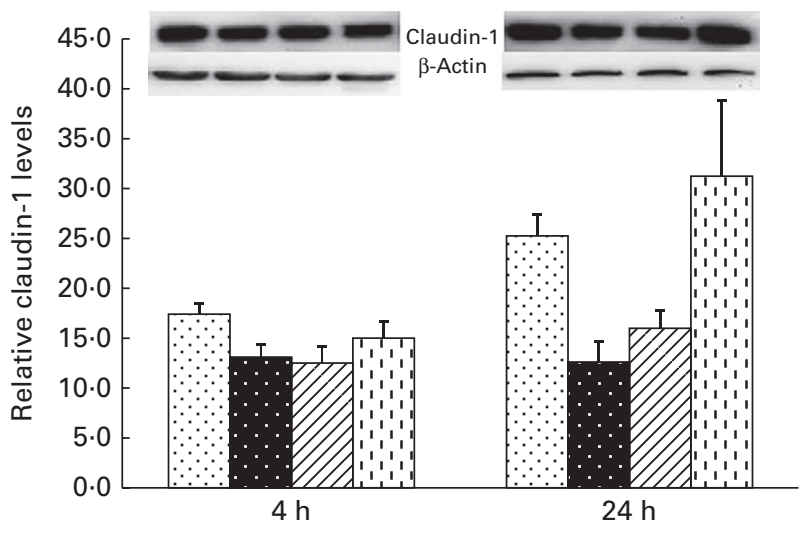

Fig. 3. Effects of asparagine (Asn) supplementation on protein expression of claudin-1 in liver after 4 or $24 \mathrm{~h}$ Escherichia coli lipopolysaccharide (LPS) challenge in weaned pigs. Relative level of claudin-1 was expressed as claudin-1 protein: $\beta$-actin protein ratio. Values are means $(n 6)$, with their standard errors represented by vertical bars. Non-challenged control (CONTR (G); pigs received a control diet and were injected with $0.9 \%$ sterile saline; LPSchallenged control (LPSCC ( $\mathbf{0}$ ); pigs received the same control diet and were challenged with LPS); LPS $+0.5 \%$ Asn ( $\square$, pigs received a $0.5 \%$ Asn diet and were challenged with LPS); LPS $+1.0 \%$ Asn (口, pigs received a $1.0 \%$ Asn diet and were challenged with LPS). LPSCC $v$. CONTR was used to determine the response to LPS challenge. Linear (L) and quadratic ( $Q$ ) polynomial contrasts were used to determine the response to Asn supplementation among LPS-challenged piglets. A trend for treatment $\times$ time interaction was observed for protein expression of liver claudin-1 $(P=0.065)$. At $4 \mathrm{~h}$ : LPSCC $v$. CONTR $(P=0.030), \mathrm{L}(P=0.390), \mathrm{Q}(P=0.518)$ and at $24 \mathrm{~h}$ : LPSCC $v$. CONTR $(P=0.002), \mathrm{L}(P=0.012), \mathrm{Q}(P=0.029)$. 
that LPS stimulates Kupffer cells to secrete pro-inflammatory cytokines, especially TNF- $\alpha$, the critical mediator of liver damage ${ }^{(5)}$. Our previous research showed that dietary supplementation of 0.5 and $1.0 \%$ Asn alleviated growth suppression of weaned piglets after LPS challenge ${ }^{(24)}$. So, in the present study, we took advantage of the model for inducing liver injury by injecting LPS, to investigate whether 0.5 and $1.0 \%$ Asn supplementation could alleviate liver injury in weaned pigs.

In clinical practice, various biochemical tests are used to index liver injury, such as blood ALT, AST, GGT and AKP activities, and ALT:AST ratio ${ }^{(28,29)}$. In the present study, with increasing Asn supplementation, serum AST and AKP activities were found decreased linearly and quadratically in LPS-challenged pigs at $24 \mathrm{~h}$ post-injection. Asn also increased serum ALT activity and ALT:AST ratio linearly and quadratically. These results were also supported by histological observations; Asn alleviated severe hepatic injury such as hepatocyte caryolysis, karyopycnosis and inflammatory cell infiltration caused by LPS challenge. Therefore, these results indicate that Asn supplementation attenuated the damage of hepatic architecture and function caused by LPS challenge. Until now, research related to Asn modulation of liver health was very limited. We speculate that the protective effect of Asn on liver might be due to the following mechanisms. Asn can be readily converted into aspartate in response to immunological challenges. Aspartate enters the urea cycle, and the amino group from aspartate is required for the recycling of the citrulline into arginine in activated macrophages ${ }^{(15,30)}$. Many studies have shown that arginines maintain the hepatic

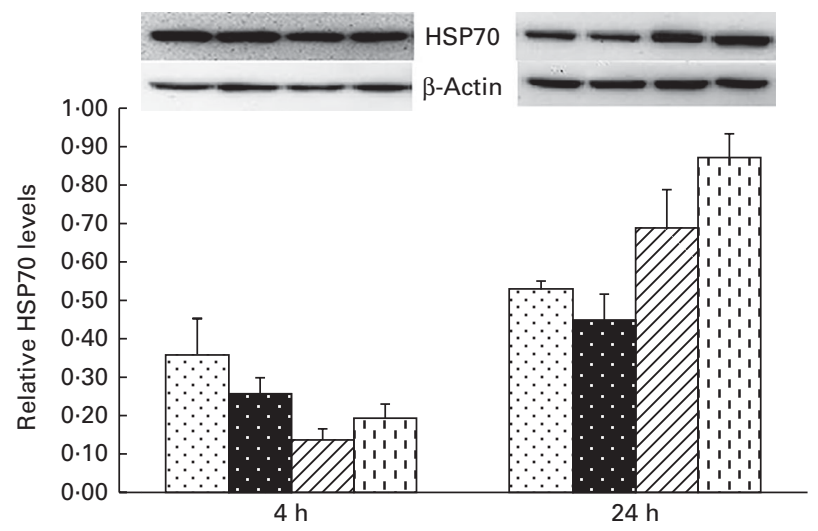

Fig. 4. Effects of asparagine (Asn) supplementation on protein expression of heat shock protein 70 (HSP70) in liver after 4 or $24 \mathrm{~h}$ Escherichia coli lipopolysaccharide (LPS) challenge in weaned pigs. Relative level of HSP70 was expressed as HSP70: $\beta$-actin protein ratio. Values are means $(n 6)$, with their standard errors represented by vertical bars. Non-challenged control (CONTR (G); pigs received a control diet and were injected with $0.9 \%$ sterile saline); LPS-challenged control (LPSCC ( $\square$ ); pigs received the same control diet and were challenged with LPS); LPS $+0.5 \%$ Asn ( $\square$, pigs received a $0.5 \%$ Asn diet and were challenged with LPS); LPS $+1.0 \%$ Asn (回, pigs received a $1.0 \%$ Asn diet and were challenged with LPS). LPSCC $v$. CONTR was used to determine the response to LPS challenge. Linear $(L)$ and quadratic $(Q)$ polynomial contrasts were used to determine the response to Asn supplementation among LPS-challenged piglets. There was treatment $\times$ time interaction for protein expression of liver HSP70 $(P<0.001)$. At $4 \mathrm{~h}$ : LPSCC v. CONTR $(P=0.337), \mathrm{L}(P=0.275), \mathrm{Q}(P=0.098)$ and at $24 \mathrm{~h}$ : LPSCC $v$. CONTR $(P=0.291), \mathrm{L}(P=0.001), \mathrm{Q}(P=0.006)$. 
integrity, and exert a protective effect in many liver injury models ${ }^{(25,31)}$. The carbon skeleton from aspartate leaves the urea cycle as fumarate which can participate in the citric acid cycle, and it increases cellular energy production ${ }^{(30)}$. In addition, aspartate is important for other aminotransferase reactions, purine and pyrimidine nucleotides synthesis, and shuttling between cytosol and the mitochondrion for gluconeogenesis, besides a role for the urea cycle ${ }^{(15)}$.

The tight junction is a pivotal cellular component for maintenance of tissue integrity, and it forms the continuous intercellular barrier against pathogen invasion. Deregulation and functional abnormality of tight junctions result in the development of liver disease ${ }^{(32,33)}$. Claudins are considered as the main constituents of the tight junction and important regulators of paracellular permeability. Consistent with the improved hepatic architecture and function, liver claudin-1 protein expression was found increased linearly and quadratically with increasing Asn supplementation at $24 \mathrm{~h}$ after LPS challenge, which suggests that Asn may improve liver integrity partially via improving the expression of liver tight junction proteins.

We hypothesised that Asn enhanced hepatic integrity by regulating hepatic inflammatory response. Increased level of TNF- $\alpha$ is an important marker of inflammation ${ }^{(3,34)}$. Several instances of evidence have demonstrated that overproduction of pro-inflammatory mediators (especially TNF- $\alpha$ ) results in liver injury ${ }^{(3,35)}$. HSP have mostly been regarded as intracellular molecules that mediate a range of essential housekeeping and cytoprotective functions ${ }^{(36)}$. High level of intracellular HSP70 can reduce the inflammatory response and improve liver regeneration ${ }^{(37)}$. Intracellular HSP70 has been shown to directly interact with the transcription factor $\mathrm{NF}-\mathrm{\kappa B}$, and prevent its activation ${ }^{(38)}$. In our present study, at $4 \mathrm{~h}$ after LPS challenge, consistent with histological and biochemical alterations in liver, LPS increased hepatic TNF- $\alpha$ mRNA and protein abundances and $H S P 7 O$ mRNA abundance. It is possible that, LPS increased the expression of proinflammatory mediators (e.g. TNF- $\alpha$ ), which further induced the expression of anti-inflammatory mediators (e.g. intracellular HSP70) by a negative feedback. With increasing Asn supplementation, mRNA abundances of TNF- $\alpha$ and HSP 70 were decreased linearly and quadratically, and protein abundance of HSP70 tended to be decreased linearly and quadratically among the LPS-challenged pigs. Surprisingly, further ELISA analysis showed that liver TNF- $\alpha$ protein concentration was not affected by Asn supplementation at $4 \mathrm{~h}$ after LPS challenge. A recent research investigating the correlation of mRNA and protein expressions has shown that mRNA and protein are differentially expressed ${ }^{(39)}$. These results indicate that, in early stage of LPS challenge, Asn might attenuate hepatic injury by decreasing the mRNA expression of pro-inflammatory mediators.

However, interestingly, at $24 \mathrm{~h}$ after LPS challenge, LPS challenge reduced mRNA expression of hepatic $T N F-\alpha$, and $T N F-\alpha$ mRNA expression was found increased linearly and quadratically with increasing Asn supplementation in the LPS-challenged pigs. Several studies have shown that Kupffer cells also have the potential to exert stimulatory influences on 
Table 4. Effects of asparagine (Asn) supplementation on mRNA abundance of Toll-like receptor 4 (TLR4) and nucleotide-binding oligomerisation domain protein (NOD) and their downstream signalling molecules after 4 or $24 \mathrm{~h}$ Escherichia coli lipopolysaccharide (LPS) challenge in weanling piglets (Mean values with their pooled standard errors, $n 6$ )

\begin{tabular}{|c|c|c|c|c|c|c|c|c|c|c|c|c|}
\hline \multirow[b]{2}{*}{ Item } & \multirow[b]{2}{*}{$\mathrm{Ti}$} & \multicolumn{4}{|c|}{$\operatorname{Tr}$} & \multirow[b]{2}{*}{ Pooled SEM } & \multicolumn{3}{|c|}{$P$} & \multicolumn{3}{|c|}{$P^{*}$} \\
\hline & & CONTR & LPSCC & LPS $+0.5 \%$ Asn & LPS $+1.0 \%$ Asn & & $\operatorname{Tr}$ & $\mathrm{Ti}$ & $\mathrm{Tr} \times \mathrm{Ti}$ & LPSCC $v$. CONTR & Linear & Quadratic \\
\hline \multirow[t]{2}{*}{ TLR4 } & $4 \mathrm{~h}$ & 1.00 & $3 \cdot 15$ & 1.97 & 1.96 & 0.30 & 0.003 & $<0.001$ & $<0.001$ & $<0.001$ & 0.015 & 0.046 \\
\hline & $24 \mathrm{~h}$ & 1.00 & 0.59 & 0.65 & 0.90 & 0.07 & & & & 0.010 & 0.011 & 0.003 \\
\hline \multirow[t]{2}{*}{ MyD88 } & $4 \mathrm{~h}$ & 1.00 & 2.87 & 2.59 & 2.65 & 0.25 & $<0.001$ & $<0.001$ & $<0.001$ & $<0.001$ & 0.506 & 0.767 \\
\hline & $24 \mathrm{~h}$ & 1.00 & 0.71 & 0.97 & 0.95 & 0.07 & & & & 0.038 & 0.001 & 0.003 \\
\hline \multirow[t]{2}{*}{ IRAK1 } & $4 \mathrm{~h}$ & 1.00 & 1.80 & $1 \cdot 11$ & 1.02 & $0 \cdot 16$ & 0.133 & $<0.001$ & $<0.001$ & 0.006 & 0.003 & 0.011 \\
\hline & $24 \mathrm{~h}$ & 1.00 & 0.60 & 0.72 & 0.86 & 0.09 & & & & 0.044 & 0.004 & 0.010 \\
\hline \multirow[t]{2}{*}{ TRAF6 } & $4 \mathrm{~h}$ & 1.00 & 1.50 & 0.98 & 0.90 & $0 \cdot 15$ & 0.180 & $<0.001$ & 0.005 & 0.056 & 0.005 & 0.023 \\
\hline & $24 \mathrm{~h}$ & 1.00 & 0.47 & 0.52 & 0.67 & 0.08 & & & & 0.089 & 0.017 & 0.017 \\
\hline \multirow[t]{2}{*}{ NOD1 } & $4 \mathrm{~h}$ & 1.00 & $2 \cdot 86$ & 1.93 & 1.80 & 0.27 & 0.042 & $<0.001$ & $<0.001$ & 0.003 & 0.015 & 0.055 \\
\hline & $24 \mathrm{~h}$ & 1.00 & 0.41 & 0.58 & 0.59 & 0.15 & & & & 0.088 & 0.037 & 0.107 \\
\hline \multirow[t]{2}{*}{ NOD2 } & $4 \mathrm{~h}$ & 1.00 & $12 \cdot 7$ & 7.85 & 7.84 & 0.73 & $<0.001$ & $<0.001$ & $<0.001$ & $<0.001$ & $<0.001$ & 0.001 \\
\hline & $24 \mathrm{~h}$ & 1.00 & 0.45 & 0.59 & 0.77 & 0.11 & & & & 0.041 & $<0.001$ & $<0.001$ \\
\hline \multirow[t]{2}{*}{ RIPK2 } & $4 \mathrm{~h}$ & 1.00 & $8 \cdot 40$ & $5 \cdot 24$ & $6 \cdot 89$ & 0.82 & $<0.001$ & $<0.001$ & $<0.001$ & 0.002 & 0.131 & 0.088 \\
\hline & $24 \mathrm{~h}$ & 1.00 & 0.50 & 0.65 & 0.78 & 0.11 & & & & 0.027 & 0.012 & 0.037 \\
\hline \multirow{2}{*}{ NF-кB p65 } & $4 \mathrm{~h}$ & 1.00 & 2.09 & 1.27 & 1.40 & 0.19 & 0.032 & $<0.001$ & $<0.001$ & 0.006 & 0.005 & 0.010 \\
\hline & $24 \mathrm{~h}$ & 1.00 & 0.70 & 0.73 & 0.82 & 0.13 & & & & 0.074 & 0.149 & 0.230 \\
\hline
\end{tabular}

Tr, Treatment; Ti, Time; CONTR, non-challenged control (piglets received a control diet and were injected with $0.9 \%$ sterile saline); LPSCC, LPS-challenged control (piglets received the same control diet and were injected with Escherichia coli LPS); LPS + 0.5\% Asn, piglets received a $0.5 \%$ Asn diet and were injected with LPS; LPS + 1.0\% Asn, piglets received a 1.0\% Asn diet and were injected with LPS; TLR4, Toll-like receptor 4; MyD88, myeloid

differentiation factor 88; IRAK1, IL-1 receptor-associated kinase 1; TRAF6, TNF- $\alpha$ receptor-associated factor 6; RIPK2, receptor-interacting serine/threonine-protein kinase 2.
* LPSCC $v$. CONTR was used to determine the response to LPS challenge. Linear and quadratic polynomial contrasts were used to determine the response to Asn supplementation among LPS-challenged piglets. 
hepatocyte regeneration by producing many kinds of cytokines, such as IL-6 and TNF- $\alpha^{(40,41)}$. Bradham et al. ${ }^{(5)}$ reported that a low expression of TNF- $\alpha$ is not beneficial, especially for hepatic regeneration. In addition, Akerman et al. ${ }^{(42)}$ reported that hepatocyte DNA synthesis was inhibited after partial hepatectomy in rats pretreated with TNF- $\alpha$ antibody. Moreover, Yang et $a l .{ }^{(43)}$ reported that some inflammatory factors might contribute to hepatic damage in early stage of liver injury, but could enhance liver regeneration in late stage, and prolonged anti-inflammatory treatment in late stage was not beneficial for hepatocyte regeneration. Mounting evidence suggests that HSP70 is also released from stressed cells as an extracellular protein that can serve as a paracrine signal. Extracellular HSP70 are capable of inducing the expression of inflammatory cytokines IL- 6 and TNF- $\alpha$, both of which are considered integral to liver regeneration ${ }^{(44)}$. Shi et $a l .{ }^{(36)}$ found that hepatic protein expression of HSP70 increased at the early phase of liver regeneration $(24 \mathrm{~h}$ after partial hepatectomy), and declined to the constitutively low level later, which indicated that HSP70 played an important role in liver regeneration. In our present study, at $24 \mathrm{~h}$ after LPS challenge, liver HSP70 protein expression was found increased linearly and quadratically with increasing Asn supplementation in the LPS-challenged pigs. These results indicate that, in late stage of LPS challenge, Asn might improve hepatocyte regeneration, by increasing the mRNA expression of $T N F-\alpha$ and the protein expression of liver HSP70. Collectively, in early and late stages of LPS challenge, Asn exerts different regulatory effects on the protein expression of hepatic HSP70 and mRNA expression of TNF- $\alpha$, and improves liver integrity.

In animal tissues, $\mathrm{NO}$ is generated enzymatically by synthases (NOS), which oxidises L-arginine to L-citrulline ${ }^{(31)}$. There are three isoforms of NOS. Of them, iNOS is present in various cell types upon pro-inflammatory cytokine stimulation. In macrophages, monocytes and other cells, the induction of iNOS and the presence of L-arginine are sufficient to initiate the generation of $\mathrm{NO}$, which have been shown to facilitate the process of hepatic regeneration ${ }^{(41,45,46)}$. In our present study, LPS reduced iNOS activities, and iNOS activity was found increased linearly and quadratically with increasing Asn supplementation in the LPS-challenged pigs. These results indicate that Asn may improve liver regeneration partially via improving iNOS activity.

To elucidate the molecular mechanism(s) by which Asn modulated hepatic inflammatory response, we investigated the role of two inflammatory signalling pathways, including transmembrane TLR and intracellular $\mathrm{NOD}^{(47)}$. Among the TLR family, TLR 4 is the most studied member which has been detected on all types of liver cells including Kupffer cells ${ }^{(8,48)}$. When TLR4 binds its ligand LPS, the TLR4/myeloid differentiation protein $2 /$ cluster of differentiation 14 receptor complex transduces a signal sensed by MyD88, which recruits IRAK to activate TRAF6, and then further activates the IкB kinase (IKK) complex. The activated IKK phosphorylates the I $\mathrm{KB}$ family, and leads ultimately to the activation of $\mathrm{NF}-\mathrm{KB}^{(48)}$. Among the NOD family, NOD1 and NOD2 are the best-characterised members, and are also highly expressed 
in liver cells. Similar to TLR4, NOD1 and NOD2 can also activate $\mathrm{NF}-\kappa \mathrm{B}$ via their adaptor molecule, RIPK2. Activation of NF- $\kappa \mathrm{B}$ results in expression of the inflammatory genes, such as proinflammatory cytokines. In the present experiment, consistent with the changes of mRNA abundance of pro-inflammatory mediators, we also observed that hepatic mRNA abundances of TLR4 signalling-related genes (TLR4, MyD88, IRAK1 and TRAFO), NOD signalling-related genes (NOD1, NOD2 and $R I P K 2)$ and $N F-\kappa B p 65$ were found decreased linearly or quadratically at $4 \mathrm{~h}$ post-injection; they were found increased linearly or quadratically at $24 \mathrm{~h}$ post-injection, with increasing Asn supplementation in the LPS-challenged pigs. So, it is possible that the beneficial effects of Asn on hepatic integrity were closely related to down-regulating mRNA expressions of TLR4 and $N O D$ signalling-related genes in early stage of LPS challenge, and up-regulating mRNA expressions of TLR 4 and NOD signalling-related genes in late stage.

Activation of TLR4 and NOD signalling triggers the production of pro-inflammatory cytokines and inflammatory response, which is crucial for host-defensive responses against invading pathogens. However, the aberrant activation of TLR4 and NOD signalling also can elicit collateral host-tissue injury $^{(49,50)}$. To prevent excessive and harmful inflammatory responses, TLR 4 and NOD signalling are negatively controlled by multiple mechanisms. Among them, negative regulators of TLR4 and NOD signalling play an important role in this process $^{(50)}$. To date, many negative regulators have been identified and characterised $^{(49,50)}$. Of them, Tollip, RP105, SOCS1 and SIGIRR are typical negative regulators of TLR 4 signalling, and ERBB2IP and CENTB1 are typical negative regulators of NOD signalling.

In our present study, at $4 \mathrm{~h}$ post-injection, LPS challenge decreased mRNA abundances of liver SIGIRR and CENTB1, and increased mRNA abundance of liver SOCS1. Similarly, Ueno-Shuto et al. ${ }^{(51)}$ reported that LPS can decrease SIGIRR expression via TLR4-p38 pathway in non-epithelial innate immune cells. In addition, Fujimoto \& Naka ${ }^{(52)}$ reported that excessive stimulation with cytokines or TLR ligands increased the expression of SOCS1 in liver, and SOCS1 expression in the liver prevented fatal hepatitis via the suppression of exacerbated liver inflammation. These results indicate that, in early phase of LPS challenge, loss of some negative regulators (SIGIRR and CENTB1) of TLR and NOD led to hyper-activation of TLR signalling. To maintain homeostasis, other negative regulators (SOCS1) might be increased to suppress overactivation of inflammatory responses ${ }^{(49)}$.

At $24 \mathrm{~h}$ post-injection, LPS decreased mRNA abundances of liver RP105, SOCS1, ERBB2IP and CENTB1, which is consistent with the decreased liver mRNA abundances of TLR 4 and $N O D$ signalling-related genes. It is possible that, in late stage of LPS challenge, the decreased mRNA expressions of TLR4 and $N O D$ negative regulators is due to low activation of TLR4 and NOD signalling pathways in LPS-challenged pigs. With increasing Asn supplementation, mRNA expressions of liver RP105, SIGIRR, ERBB2IP and CENTB1 were up-regulated linearly and quadratically, which is consistent with the up-regulated liver mRNA expressions of TLR4 and NOD signalling-related genes at $24 \mathrm{~h}$ post-injection. It is possible that, in LPS-challenged pig fed the Asn diet, the increased mRNA expressions of TLR4 and NOD signalling-related genes induced the increased mRNA expressions of their negative regulators in a negative feedback.

In addition, with increasing Asn supplementation, mRNA expression of liver Tollip was found increased linearly and quadratically in the LPS-challenged pigs. High level of Tollip can suppress overactivation of inflammatory response caused by TLR4 signalling ${ }^{(53)}$. So, it is possible that Asn attenuated hepatic injury partially via improving the mRNA expression of Tollip.

In our present study, dynamic changes occur in proinflammatory gene expression in liver after LPS challenge ${ }^{(54)}$. So, measurements taken only in early and late stages ( 4 and $24 \mathrm{~h})$ are probably not adequate. In addition, only two levels of Asn supplemented ( 0.5 and $1.0 \%$ Asn) were used in our present study. Compared with lower concentration of Asn $(0.5 \%)$, higher concentration $(1.0 \%)$ had better effect on liver morphology, serum biochemical parameters, claudin-1 and HSP70 protein expressions, mRNA abundance of hepatic TLR4 and NOD signalling-related genes and their negative regulators, especially at $24 \mathrm{~h}$ after LPS challenge, but it had almost the same effect on other data in our present study. So, $1.0 \%$ Asn supplement might not be the most appropriate dose. If we had had more doses of Asn, the most appropriate doses would have been obtained for improving liver integrity, for the quantification of which further experiments are necessitated. Moreover, measurements only for mRNA abundances of $T L R$ and $N O D$ signalling-related molecules (for example, TLR4, NOD and $N F-\kappa B$ p65 mRNA expressions) are not sufficient to confirm the roles of these signalling molecules in liver injury. Thus, in future studies, further measurements such as of TLR4 or NOD activation and NF-кB nuclear translocation at more time points are also needed to understand better their roles in LPS-induced liver injury.

In conclusion, Asn supplementation improves liver integrity in LPS-challenged piglets. In addition, Asn inhibits the expressions of hepatic pro-inflammatory mediators, and TLR4 and NOD signalling-related genes in early stage of LPS challenge, and increases NOS activity, HSP70 synthesis and the expressions of hepatic pro-inflammatory mediators, TLR4 and NOD signalling-related genes and their negative regulators in late stage.

\section{Supplementary material}

To view supplementary material for this article, please visit http://dx.doi.org/10.1017/S0007114515001476

\section{Acknowledgements}

The present study was supported by the National Natural Science Foundation of China (31422053, 31372318 and 31172222 ) and the Project of Natural Science Foundation of Hubei Province (2013CFA029).

The authors' contributions are as follows: Y. L. contributed to the study design, participated in the statistical analysis 
and interpretation of data, and writing of the paper; H. W. collected data, participated in the statistical analysis and interpretation of data, and writing of the paper; D. P. and W. L. collected data and participated in the statistical analysis; H. Z. contributed to the study design, participated in the statistical analysis and interpretation of data; Y. H., S. L., H. S. and $\mathrm{X}$. W. contributed to data collection; Y. L. had primary responsibility for the final content. All authors read and approved the final version of the manuscript.

The authors declare that they have no conflict of interest.

\section{References}

1. Gao B, Jeong WI \& Tian Z (2008) Liver: an organ with predominant innate immunity. Hepatology 47, 729-736.

2. Racanelli V \& Rehermann B (2006) The liver as an immunological organ. Hepatology 43, 2 Suppl. 1, S54-S62.

3. Chen F, Liu Y, Zhu H, et al. (2013) Fish oil attenuates liver injury caused by LPS in weaned pigs associated with inhibition of TLR 4 and nucleotide-binding oligomerization domain protein signaling pathways. Innate Immun 19, 504-515.

4. Schmöcker C, Weylandt KH, Kahlke L, et al. (2007) Omega-3 fatty acids alleviate chemically induced acute hepatitis by suppression of cytokines. Hepatology 45, 864-869.

5. Bradham CA, Plümpe J, Manns MP, et al. (1998) Mechanisms of hepatic toxicity. I. TNF-induced liver injury. Am J Physiol 275, G387-G392.

6. Fukata M, Vamadevan AS \& Abreu MT (2009) Toll-like receptors (TLRs) and Nod-like receptors (NLRs) in inflammatory disorders. Semin Immunol 21, 242-253.

7. Takeuchi O \& Akira S (2010) Pattern recognition receptors and inflammation. Cell 140, 805-820.

8. Szabo G, Dolganiuc A \& Mandrekar P (2006) Pattern recognition receptors: a contemporary view on liver diseases. Hepatology 44, 287-298.

9. Schwabe RF, Seki E \& Brenner DA (2006) Toll-like receptor signaling in the liver. Gastroenterology 130, 1886-1900.

10. Moreira LO \& Zamboni DS (2012) NOD1 and NOD2 signaling in infection and inflammation. Front Immunol 3, 328.

11. Scott MJ, Chen C, Sun Q, et al. (2010) Hepatocytes express functional NOD1 and NOD2 receptors: a role for NOD1 in hepatocyte CC and CXC chemokine production. I Hepatol 53, 693-701.

12. Swain B, Basu M \& Samanta M (2013) NOD1 and NOD2 receptors in mrigal (Cirrbinus mrigala): inductive expression and downstream signalling in ligand stimulation and bacterial infections. $J$ Biosci 38, 533-548.

13. Wu G (2009) Amino acids: metabolism, functions, and nutrition. Amino Acids 37, 1-17.

14. Wu G, Bazer FW, Davis TA, et al. (2007) Important roles for the arginine family of amino acids in swine nutrition and production. Livest Sci 112, 8-22.

15. Li P, Yin YL, Li D, et al. (2007) Amino acids and immune function. Br J Nutr 98, 237-252.

16. Suzuki F, Okayasu H, Tashiro M, et al. (2002) Effect of lignins and their precursors on nitric oxide, citrulline and asparagine production by mouse macrophage-like Raw 264.7 cells. Anticancer Res 22, 2719-2724.

17. Suzuki F, Tashiro M, Hashimoto K, et al. (2002) Stimulation of arginine consumption and asparagine production in LPS-activated macrophages. Anticancer Res 22, 203-209.

18. Chen T, Zamora R, Zuckerbraun B, et al. (2003) Role of nitric oxide in liver injury. Curr Mol Med 3, 519-526.
19. Ito Y, Abril ER, Bethea NW, et al. (2004) Role of nitric oxide in hepatic microvascular injury elicited by acetaminophen in mice. Am J Physiol Gastrointest Liver Physiol 286, G60-G67.

20. Puiman P \& Stoll B (2008) Animal models to study neonatal nutrition in humans. Curr Opin Clin Nutr Metab Care 11, 601-606.

21. Merrifield CA, Lewis M, Claus SP, et al. (2011) A metabolic system-wide characterisation of the pig: a model for human physiology. Mol Biosyst 7, 2577-2588.

22. National Research Council (1998) Nutrient Requirements of Swine, 10th ed. Washington, DC: National Academic Press.

23. Wu G, Davis PK, Flynn NE, et al. (1997) Endogenous synthesis of arginine plays an important role in maintaining arginine homeostasis in postweaning growing pigs. $J$ Nutr 127, 2342-2349.

24. Li S, Liu YL, Shi HF, et al. (2012) Effect of asparagine acid on growth performance, blood cell differential count, and blood biochemical measurements of weaned piglets after lipopolysaccharide challenge. Chinese J Anim Nutr 24, 2450-2458.

25. Li Q, Liu Y, Che Z, et al. (2012) Dietary L-arginine supplementation alleviates liver injury caused by Escherichia coli LPS in weaned pigs. Innate Immun 18, 804-814.

26. Livak KJ \& Schmittgen TD (2001) Analysis of relative gene expression data using real-time quantitative PCR and $2^{-\Delta \Delta \mathrm{CT}}$ method. Methods 25, 402-408.

27. Koenderman L, Buurman W \& Daha MR (2014) The innate immune response. Immunol Lett 162, 95-102.

28. Lee K \& Yang JH (2013) Which liver enzymes are better indicators of metabolic syndrome in adolescents: the Fifth Korea National Health and Nutrition Examination Survey, 2010. Metab Syndr Relat Disord 11, 229-235.

29. Van Beek JH, de Moor MH, de Geus EJ, et al. (2013) The genetic architecture of liver enzyme levels: GGT, ALT and AST. Behav Genet 43, 329-339.

30. Adam J, Yang M, Bauerschmidt C, et al. (2013) A role for cytosolic fumarate hydratase in urea cycle metabolism and renal neoplasia. Cell Rep 3, 1440-1448.

31. Wu G, Bazer FW, Davis TA, et al. (2009) Arginine metabolism and nutrition in growth, health and disease. Amino Acids $\mathbf{3 7}$, 153-168.

32. Lee NP \& Luk JM (2010) Hepatic tight junctions: from viral entry to cancer metastasis. World J Gastroenterol 16, 289-295.

33. Anderson JM \& Van Itallie CM (2009) Physiology and function of the tight junction. Cold Spring Harb Perspect Biol $\mathbf{1}$, a002584.

34. Jacobi SK, Moeser AJ, Corl BA, et al. (2012) Dietary longchain PUFA enhance acute repair of ischemia-injured intestine of suckling pigs. $J$ Nutr 142, 1266-1271.

35. Kanuri G, Spruss A, Wagnerberger S, et al. (2011) Role of tumor necrosis factor $\alpha(\mathrm{TNF} \alpha)$ in the onset of fructoseinduced nonalcoholic fatty liver disease in mice. $J$ Nutr Biochem 22, 527-534.

36. Shi Q, Dong Z \& Wei H (2007) The involvement of heat shock proteins in murine liver regeneration. Cell Mol Immunol 4, 53-57.

37. Oka Y, Akagi Y, Kinugasa T, et al. (2013) Heat-shock pretreatment reduces liver injury and aids liver recovery after partial hepatectomy in mice. Anticancer Res 33, 2887-2894.

38. Kuboki S, Schuster R, Blanchard J, et al. (2007) Role of heat shock protein 70 in hepatic ischemia-reperfusion injury in mice. Am J Physiol Gastrointest Liver Physiol 292, G1141-G1149.

39. Anderson L \& Seilhamer J (1997) A comparison of selected mRNA and protein abundances in human liver. Electrophoresis 18, 533-537. 
40. Malik R, Selden C \& Hodgson H (2002) The role of nonparenchymal cells in liver growth. Semin Cell Dev Biol 13, 425-431.

41. Yokoyama Y, Nagino M \& Nimura Y (2007) Mechanism of impaired hepatic regeneration in cholestatic liver. $J$ Hepatobiliary Pancreat Surg 14, 159-166.

42. Akerman P, Cote P, Yang SQ, et al. (1992) Antibodies to tumor necrosis factor- $\alpha$ inhibit liver regeneration after partial hepatectomy. Am J Physiol 263, G579-G585.

43. Yang R, Zou X, Koskinen ML, et al. (2012) Ethyl pyruvate reduces liver injury at early phase but impairs regeneration at late phase in acetaminophen overdose. Crit Care 16, R9.

44. Wolf JH, Bhatti TR, Fouraschen S, et al. (2014) Heat shock protein 70 is required for optimal liver regeneration after partial hepatectomy in mice. Liver Transpl 20, 376-385.

45. Guzik TJ, Korbut R \& Adamek-Guzik T (2003) Nitric oxide and superoxide in inflammation and immune regulation. J Physiol Pharmacol 54, 469-487.

46. Malyshev IY, Bayda LA, Trifonov AI, et al. (2000) Cross-talk between nitric oxide and HSP70 in the antihypotensive effect of adaptation to heat. Physiol Res 49, 99-105.

47. Al-Sayeqh AF, Loughlin MF, Dillon E, et al. (2010) Campylobacter jejuni activates NF-KB independently of TLR2, TLR4, Nod1 and Nod2 receptors. Microb Pathog 49, 294-304.
48. Becker CE \& O'Neill LA (2007) Inflammasomes in inflammatory disorders: the role of TLRs and their interactions with NLRs. Semin Immunopathol 29, 239-248.

49. Kondo T, Kawai T \& Akira S (2012) Dissecting negative regulation of Toll-like receptor signaling. Trends Immunol 33, 449-458.

50. Coll RC \& O'Neill LA (2010) New insights into the regulation of signalling by toll-like receptors and nod-like receptors. I Innate Immun 2, 406-421.

51. Ueno-Shuto K, Kato K, Tasaki Y, et al. (2014) Lipopolysaccharide decreases single immunoglobulin interleukin-1 receptor-related molecule (SIGIRR) expression by suppressing specificity protein 1 (Sp1) via the Toll-like receptor 4 (TLR4)-p38 pathway in monocytes and neutrophils. $J$ Biol Chem 289, 18097-18109.

52. Fujimoto M \& Naka T (2010) SOCS1, a negative regulator of cytokine signals and TLR responses, in human liver diseases. Gastroenterol Res Pract pii, 470468.

53. Steenholdt C, Andresen L, Pedersen G, et al. (2009) Expression and function of toll-like receptor 8 and Tollip in colonic epithelial cells from patients with inflammatory bowel disease. Scand J Gastroenterol 44, 195-204.

54. Xu FL, You HB, Li XH, et al. (2008) Glycine attenuates endotoxin-induced liver injury by downregulating TLR4 signaling in Kupffer cells. Am J Surg 196, 139-148. 